\title{
Anatomic Variation of the Internal Oblique Muscle Detected during Transversus Abdominis Plane Block
}

\author{
Umit Yasar Tekelioglu ${ }^{1^{*}}$, Abdullah Demirhan ${ }^{1}$, Tülay Özlü ${ }^{2}$ and Hasan Kocoglu ${ }^{1}$ \\ ${ }^{1}$ Department of Anesthesiology and Reanimation, Abant Izzet Baysal University Medical School, Bolu, Turkey \\ ${ }^{2}$ Department of Obstetrics and Gynecology, Abant Izzet Baysal University Medical School, Bolu, Turkey
}

"Corresponding author: Umit Yasar Tekelioglu, MD, Department of Anesthesiology and Reanimation, Abant Izzet Baysal University Medical School, Bolu, Turkey, Tel: +90 505 3131292; Fax: +90 374 2534615; E-mail: drtekelioglu@yahoo.com

Rec date: Nov 25, 2014 Acc date: June 10, 2015 Pub date: June 15, 2015

Copyright: (c) 2015 Tekelioglu UY, et al. This is an open-access article distributed under the terms of the Creative Commons Attribution License, which permits unrestricted use, distribution, and reproduction in any medium, provided the original author and source are credited.

\section{Case Report}

We report a 35 years-old female patient with a body mass index of $29.7 \mathrm{~kg} / \mathrm{m} 2$ to whom we performed a transversus abdominis plane (TAP) block following caesarean section. Her past medical history was uneventful. The surgery was performed under general anaesthesia since she refused spinal anaesthesia. She gave written informed consent for the application of TAP block at the end of the operation for postoperative pain control. During application of TAP block under general anaesthesia and under ultrasound guidance, we came across an anatomic variation of the unilateral internal oblique muscle (Figure 1). Internal oblique muscle fascia merged with fascia of the external oblique muscle by a fibrous band which resulted with an interruption in the internal oblique muscle. We carefully injected the local anaesthetic agent to the neurofascial anatomic space between the internal oblique muscle and transversus abdominus muscle. Patient was extubated uneventfully at the end of procedure.

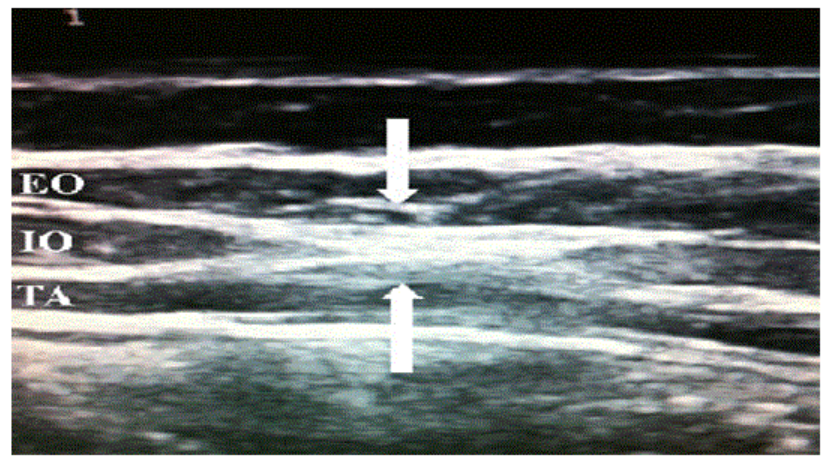

Figure 1: The ultrasound image demonstrates the fibrous adhesion between the fascia of the internal oblique and external oblique muscles.

Blocking of the sensory nerves originated from the lower six thoracic and first lumbar nerve roots and producing a regional, abdominal wall nerve block can be acquired by a single TAP block procedure which involves local anesthetic injection to fascial plane between the internal oblique and transversus abdominis muscles [1]. TAP block is reported to be an effective method to reduce use of postoperative analgesics in patients undergoing caesarean section [2]. The procedure can be applied by either the classical blind technique or under ultrasound guidance $[1,3]$. In the blind technique which was first described by Rafi', the procedure is directed according to the double-pop feeling that occurs during passage of the needle through the fascias of the external and internal oblique muscles [1]. In TAP block procedures that are applied without ultrasound guidance, anatomic variations may cause confusions in feeling the expected double-pop and this may lead to injuries of the intraabdominal organs [4]. As a result of our literature search, we could meet only one case report that describes an anatomic variation of the internal oblique muscle [5].

In conclusion, TAP block procedures are frequently used for postoperative pain control nowadays. We believe that, the blind technique that does not use the guidance of ultrasound may lead to intrabdominal organ injuries in the presence of possible anatomic variations of the internal oblique and transversus abdominis muscles.

\section{References}

1. Rafi AN (2001) Abdominal field block: a new approach via the lumbar triangle. Anaesthesia 56: 1024-1026

2. Bamigboye AA, Hofmeyr GJ (2009) Local anaesthetic wound infiltration and abdominal nerves block during caesarean section for postoperative pain relief. Cochrane Database Syst Rev 3:CD006954

3. Hebbard P, Fujiwara Y, Shibata Y, Royse C (2207) Ultrasound-guided transversus abdominis plane (TAP) block. Anaesth Intensive Care 35: 616-617.

4. McDermott G, Korba E, Mata U, Jaigirdar M, Narayanan N, et al. (2012) Should we stop doing blind transversus abdominis plane blocks? Br J Anaesth 108: 499-502

5. Lew VK, Gray AT (2012) An Unusual Transversus Abdominis Plane Block: Anatomic Variation in the Internal Oblique Muscle. Anesthesiology 119: 1209. 